\title{
Myocardial abscess and sternal osteomyelitis following myocardial infarction and resuscitation
}

\author{
R.E.A. Smith, J.E. Martin and P.G. Mills \\ Departments of Cardiology and Morbid Anatomy, The London Hospital, London E1 IBB, UK.
}

\begin{abstract}
Summary: A 58 year old man presented with an anterior myocardial infarction requiring repeated resuscitation. He had impaired glucose tolerance. He died five weeks later and post-mortem examination revealed a myocardial abscess and sternal osteomyelitis due to Staphylococcus aureus. These had not been apparent clinically. The possible aetiology and difficulties in diagnosis are discussed.
\end{abstract}

\section{Introduction}

Myocardial abscess formation is a rare complication of myocardial infarction and fracture of the sternum may be the result of resuscitation. Sternal osteomyelitis is usually reported following median sternotomy. We report a case of myocardial abscess and sternal osteomyelitis following myocardial infarction and resuscitation. Problems of diagnosis are discussed. With increasing degrees of intervention after myocardial infarction the possibility of infective episodes, including unusual foci, should be considered.

\section{Case report}

A previously well 58 year old man presented with a large anterior myocardial infarction complicated initially by ventricular fibrillation and left ventricular failure. Later investigation revealed impaired glucose tolerance with a two hour concentration of $10.9 \mathrm{mmol} / \mathrm{l}$ after a $75 \mathrm{~g}$ load.

He had recurrent episodes of ventricular tachycardia for 3 weeks requiring external cardiac massage and DC shock on five occasions. He was treated with intravenous lignocaine, amiodarone and frusemide via a subclavian line and had a temporary pacing wire inserted to overpace the ventricular arrythmias.

He had a typical fever following the infarction and later a low grade intermittent fever not exceeding $37.5^{\circ} \mathrm{C}$. His white cell count was not raised. Four weeks after infarction he complained of pericardialtype chest pain. No rub was heard and the ESR was normal. Serial electrocardiograms had revealed persistent elevation of the ST segments, thought to be consistent with a left ventricular aneurysm. This was

Correspondence: J.E. Martin, M.A., M.B.

Accepted: 9 February 1989 supported by digital subtraction angiography and ultrasound, and he was anticoagulated with warfarin.

He died five weeks after infarction with progressive cardiac failure. At post-mortem an abscess cavity was found in the sternum at the site of a fracture just below the angle of Louis. The anterior wall of the left ventricle showed transmural infarction and also contained an abscess (see Figure 1). There was no anatomical communication between the two abscess cavities. The left ventricle was dilated and there was mural thrombus overlying the area of infarction. The left anterior descending and circumflex arteries showed severe atheroma with occluding thrombus.

Staphylococcus aureus was grown from the pus in the sternum and from the myocardium, the mural thrombus was sterile. No other septic foci were found.

\section{Discussion}

Myocardial abscess formation is rare and is usually associated with infective endocarditis. A recent review noted only 16 cases of abscess within the territory of a myocardial infarct. ${ }^{1}$ Most cases are related to bacteraemia following instrumentation or concurrent infection such as pneumonia. ${ }^{2}$ The majority were unexpected findings at post-mortem without antemortem clinical features. Staphylococcus aureus is one of the variety of organisms that has been isolated from such lesions. As in our case, there is a recognized association with diabetes mellitus. The incidence of this condition is very low, but may be greater than that suggested by the literature because of the difficulty in diagnosis.

Sternal fracture is a well known complication of cardiac resuscitation, occurring in up to $21 \%$ of unsuccessful cases. ${ }^{3}$ Osteomyelitis of the sternum is, however, unusual other than after median sternotomy.

The Fellowship of Postgraduate Medicine, 1989 


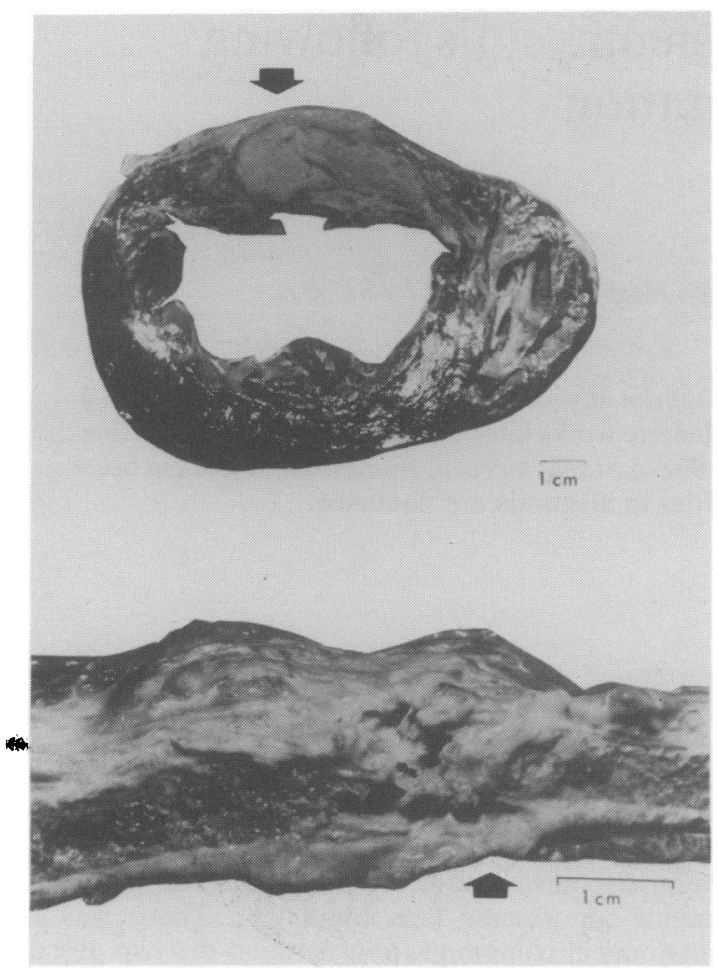

Figure 1 The upper photograph shows a horizontal section through the ventricular walls. There is an abscess cavity in the anterior wall of the left ventricle (arrow). The lower photograph shows a longitudinal section through the body of the sternum. There is an abscess cavity at a fracture site below the angle of Louis (arrow).

\section{References}

1. Richards, J. Bacteroides fragilis isolated post mortem from an abscess in a myocardial infarct. $J$ Infect 1985, 10: 268-271.

2. Vianna, N.J. Myocardial abscess and infarction. Report of a case and a review of the literature. J Med Soc New Jersey 1973, 70: 931-932.

3. Krischer, J.P., Fine, E.G., Davis, J.H. \& Nagel, E.L. Complications of cardiac resuscitation. Chest 1987, 92: 287-291.

4. Mardelli, T.J., Ogawa, S., F.E., Dreifus, L.S. \& Meixell, L.L. Cross-sectional echocardiographic detection of aortic ring abscess in bacterial endocarditis. Chest 1978, 74: 576-578.
In this case neither the clinical or radiographic findings were suggestive of abscess formation.

Both abscesses in our case are presumed to have resulted from staphylococcal bacteraemia at instrumentation. Myocardial infection may have occurred via collateral vessels supplying the infarcted territory, or seeding may have occurred via Thebesian veins. There were no specific clinical features to suggest the diagnosis in this instance.

Echocardiography has been suggested as being of use in the diagnosis of aortic ring abscess, ${ }^{4}$ but in our case an abnormality consistent with aneurysm was seen and there was no suggestion of fluid collection, even on retrospective review of the films. ECG-gated computerized tomography of the heart has also been mooted as a diagnostic technique for a range of cardiac conditions including intramyocardial tumours, ${ }^{5}$ however this technique requires specialized equipment and skills that are not as yet widely available. Gallium-67 citrate scanning has been of use in the diagnosis of septic foci, including annular ring abscess in endocarditis, but there is a suspicion that the isotope may accumulate in acutely infarcted myocardium. $^{6}$ This technique may, however, have aided the diagnosis of the sternal focus of infection, had the possibility of abscess been suspected on clinical grounds.

If low grade fever without neutrophilia occurs late following myocardial infarction, particularly in a diabetic, and instrumentation has occurred, then appropriate antimicrobial therapy should be considered.

5. Lackner, K. \& Thurn, P. Computed tomography of the heart: ECG-gated and continuous scans. Radiology 1981, 140: 413-420.

6. Kramer, R.J., Goldstein, R.E., Hirshfield, J.W. JR. et al. Accumulation of gallium-67 in regions of acute myocardial infarction. Am J Cardiol 1974, 33: 861-867. 